\title{
Localización del atributo más valorado de un cosmético antiedad con el método multivariante MAXDIFF
}

\section{Location of the most valued attribute of an anti-aging cosmetic with the multivariate MAXDIFF method}

Iván Estuardo Pazmiño Reinoso

Universidad Internacional del Ecuador, Ecuador

Autor para correspondencia: ivanpazmino1 @ hotmail.com

Fecha de recepción: 09 de Junio de 2017 - Fecha de aceptación: 30 de Septiembre de 2017

\begin{abstract}
Resumen
El objetivo de la actual investigación es localizar estadísticamente el atributo más valorado por los clientes institucionales de un cosmético antiedad de ojos en mercado institucional del Distrito metropolitano de Quito. La metodología aplicada tiene un alcance, descriptivo, transversal sobre la base de estudios de carácter cualitativo y cuantitativo. El análisis de los datos en una primera etapa se abordó recurriendo a técnicas cualitativas tradicionales como es la entrevista a profundidad y en una segunda etapa con una técnicas cuantitativas multivariante, denominada análisis MAXDIFF. Estos análisis se aplicaron a una población muestral de 96 clientes institucionales, consumidores de cosméticos antiedad cuyos resultados hicieron posible conocer cual atributo de producto prefiere este tipo de cliente. Este estudio tiene aplicaciones prácticas pues permite conocer sobre cual atributos debe dirigirse el esfuerzo de Marketing en este segmento de mercado, además de presentar una metodología de búsqueda del atributo principal inédita y que puede aplicarse a cualquier tipo de producto.
\end{abstract}

Palabras Claves: atributos; marketing; maxdiff

\begin{abstract}
The goal of current research is statistically locate the most valued by institutional clients of an antiaging cosmetic eye institutional market in the Metropolitan District of Quito attribute. The methodology has a range, transversal studies based on qualitative and quantitative. The analysis of data in a first step board using traditional techniques such qualitative depth interviews and in a second step with a quantitative multivariate techniques known MAXDIFF analysis. These analyzes were applied to a sample population of 96 institutional clients, consumers of cosmetic anti-aging and the results made it possible to know which product attribute prefer this type of client. This study has practical applications because it allows to know about which attributes should be directed marketing effort in this market segment, besides presenting a methodology search and unpublished primary attribute that can be applied to any type of product.
\end{abstract}

Key words: attributes; marketing; maxdiff 


\section{Introducción}

La técnica denominada maximum difference scaling (Maxdiff) gravita en ofrecer de una forma sencilla, a los entrevistados un conjunto de alternativas para que evalúen las mejores y las peores opciones (Villaseca, 2014), para analizar características en investigaciones de marketing. (Sreedhar, 2016), así como para "medir la importancia relativa de un conjunto de atributos para un grupo de encuestados" (Ochoa, 2014). De tal manera que se hace posible obtener una jerarquía de atributos ordenados, que van desde el más importante al menos importante (Talledo, 2015). Según los técnicos (XLSTAT, 2015), el análisis Maxdiff se utiliza para comprender los atributos clave de un producto, y es menos complicado que el análisis conjunto.

El análisis Maxdiff se hace en dos pasos. En el primero el software estadística XLSAT genera un diseño, de modo que cada atributo se presenta en relación con otros atributos, un número igual de veces (XLSTAT, 2015). Obteniendo una tabla en donde el encuestado responde cual le parece el peor y mejor atributo cada grupo, ver tabla 1. El procedimiento de llenado del diseño, consiste que el encuestado vea un grupo de atributos como puede ser ("ingredientes europeos, precio bajo, presentación de lujo "), luego el encuestado escoge de este grupo lo que a él le parece el mejor y peor atributo. Si escogió "ingredientes europeos" como el mejor atributo pondrá el número "1" en la casilla de "individuo mejor", si escogió "precio bajo" como peor atributo, pondrá el número " 2 " en la casilla de "individuo peor", haciendo notar que nunca un atributo puede ser puesto como mejor y peor a la vez. Con este mismo procedimiento se le siguen presentando al encuestado las opciones de peor y mejor atributo hasta que se termine de llenar la tabla, lo que se hizo en un tiempo estimado máximo de cinco minutos para esta investigación.

Tabla 1 Tabla de casos ponderados por frecuencia

\begin{tabular}{|c|c|c|c|c|c|c|c|c|}
\hline Elección 1 & Elección 2 & Elección 3 & \multicolumn{3}{|c|}{$\begin{array}{l}\text { Individuo } 1 \\
\text { Mejor }\end{array}$} & \multicolumn{3}{|c|}{$\begin{array}{l}\text { Individuo } 1 \\
\text { Peor }\end{array}$} \\
\hline $\begin{array}{l}\text { Ingredientes } \\
\text { europeos }\end{array}$ & Precio bajo & Presentación de lujo & 1 & 2 & 3 & 1 & 2 & 3 \\
\hline $\begin{array}{l}\text { Facilidad de } \\
\text { empleo }\end{array}$ & Suprime ojeras & $\begin{array}{l}\text { Capacitación a cliente } \\
\text { corporativo }\end{array}$ & 1 & 2 & 3 & 1 & 2 & 3 \\
\hline $\begin{array}{l}\text { Elimina las } \\
\text { bolsas de ojos }\end{array}$ & Calidad farmacéutica & $\begin{array}{l}\text { Beneficios comerciales, } \\
\text { créditos y descuentos }\end{array}$ & 1 & 2 & 3 & 1 & 2 & 3 \\
\hline Suprime ojeras & Facilidad de empleo & Precio bajo & 1 & 2 & 3 & 1 & 2 & 3 \\
\hline Erradica arrugas & Presentación de lujo & Elimina las bolsas de ojos & 1 & 2 & 3 & 1 & 2 & 3 \\
\hline $\begin{array}{l}\text { Amigable con la } \\
\text { piel }\end{array}$ & Ingredientes europeos & $\begin{array}{l}\text { Beneficios comerciales, } \\
\text { créditos y descuentos }\end{array}$ & 1 & 2 & 3 & 1 & 2 & 3 \\
\hline $\begin{array}{l}\text { Calidad } \\
\text { farmacéutica }\end{array}$ & Amigable con la piel & Suprime ojeras & 1 & 2 & 3 & 1 & 2 & 3 \\
\hline precio bajo & $\begin{array}{l}\text { Beneficios comerciales, } \\
\text { créditos y descuentos }\end{array}$ & Erradica arrugas & 1 & 2 & 3 & 1 & 2 & 3 \\
\hline $\begin{array}{l}\text { Facilidad de } \\
\text { empleo }\end{array}$ & Elimina las bolsas de ojos & Ingredientes europeos & 1 & 2 & 3 & 1 & 2 & 3 \\
\hline
\end{tabular}

\footnotetext{
${ }^{1}$ Primer grupo de la Tabla 1
} 


\begin{tabular}{lll|l|l|l|l|l|l|l|l|}
\hline $\begin{array}{l}\text { Capacitación a } \\
\text { cliente }\end{array}$ & Erradica arrugas & Amigable con la piel & 1 & 2 & 3 & 1 & 2 & 3 \\
$\begin{array}{l}\text { corporativo } \\
\begin{array}{l}\text { Presentación de } \\
\text { lujo }\end{array}\end{array}$ & $\begin{array}{l}\text { Capacitación a cliente } \\
\text { corporativo }\end{array}$ & Calidad farmacéutica & 1 & 2 & 3 & 1 & 2 & 3 \\
\hline
\end{tabular}

Nota. Fuente: Investigación cuantitativa propia del autor.

Tabla realizada con el software estadístico XLSAT 2015.

En el segundo paso se ingresan los resultados de la tabla 1 al software estadístico XLSTAT 2015 para su procesamiento y obtención de resultados.

\section{Problema a investigar}

¿Cuál es el atributo más valorado de un cosmético antiedad por el mercado institucional de centros de estética en el distrito metropolitano de Quito?

\section{Objetivos de la investigación}

Descubrir cuál es el atributo que más influye en la elección de compra de un cosmético antiedad en el mercado institucional de centros de estética en el distrito metropolitano de Quito

Preguntas que se han planteado para esta investigación. De manera explícita o implícita.

$\checkmark$ ¿Cuáles son los atributos que generan preferencia en productos antiedad?

$\checkmark \quad$ ¿Cuál es el atributo preferido por el cliente institucional?

$\checkmark$ ¿Cuáles son los atributos menos importantes de cosmético antiedad?

$\checkmark \quad$ ¿Cuál es el atributo que generaría un posicionamiento efectivo para lograr llegar al máximo número de clientes potenciales?

Justificación de la investigación. Es decir, ¿por qué esta investigación es importante? ¿Qué es lo que le distingue?

Para el autor es significativo realizar este aporte, ya que se investigó de una manera inédita y práctica las características y el comportamiento del consumidor de un nicho mercado muy específico en la ciudad de Quito. Constituyendo esta investigación una secuencia y un formato de trabajo que puede ser aplicado a varios tipos de productos o servicios de cualquier índole con una herramienta estadística multivariante. Con la información obtenida acerca del atributo más relevante se puede realizar planes estratégicos de marketing y posicionamiento en torno al atributo más valorado, aumentando las probabilidades de éxito en el mercado de un producto dado, utilizando resultados cuantitativos y no solamente cuantitativos.

Este de notar que la aplicación de la metodología de este estudio para investigación de mercados abre la posibilidad de generación de empleo, puesto que se necesitan profesionales de varias áreas para poder realizarla, y que al explotarlas, según reconocidos eruditos de la materia (Zea, 2010), se generan fuentes de riqueza que contribuyen al bienestar ciudadano, de acuerdo a la política del Buen Vivir, propulsada por el gobierno nacional del Ecuador. 


\section{Marco o perspectiva teórica o conceptual}

El propósito central de este estudio es investigar cuál es el atributo más valorado de un cosmético antiedad de ojos en el mercado institucional del Distrito Metropolitano de Quito, definiendo a un atributo como la mejor característica, función, beneficio y uso de un producto o servicio (Thompson, 2015), así mismo en este trabajo se aplicando una herramienta de análisis multivariante la cual mida, explique y prediga el grado de relación entre las combinaciones múltiples de variables, (Hair, 1999, pág. 39), que tienen que ver los atributos de elección de compra por parte del cliente institucional, esto en razón de que las herramientas estadísticas multivariantes tienen la ventaja sobre los métodos univariantes de combinar todas la variables (características) a la vez, eliminar la información redúndate y obtener una nueva variable que no es observable directamente (Martín Castellon, Lafuente Lechuga, \& Faura Martínez, 2015), sobre la cual se hacen los análisis de relación entre variables (Pérez C. , 2009).

\section{Diseño de la investigación}

Esta investigación fue realizada en dos fases:

a) Investigación cualitativa en base a 12 entrevistas a profundidad no probabilísticas, por juicio: a propietarios, administradores o funcionarios de centros de estética ubicados en el Distrito Metropolitano de Quito

b) Investigación cuantitativa en base a 96 encuestas con un nivel del confianza del $95 \%$ y un margen de error del $6 \% .{ }^{2}$ De un universo de 147 clientes institucionales y en funcionamiento en el Distrito Metropolitano de Quito, dato que fue proporcionado por uno de los laboratorios farmacéuticos ecuatorianos legalmente reconocidos, en base a su censo comercial en el Distrito Metropolitano de Quito, a diciembre del 2014, número que es similar al reportado por la prensa (El Comercio, 2012). En lo referente al análisis multivariante MAXDIFF fue realizado con el software estadístico XLSTAT, utilizando el método Hierarchical Bayes, con una Convergencia del 0.0001, con 2019454 de Iteraciones: y un tiempo de procesamiento de 7641 Segundos.

\section{Estadísticos de bondad de ajuste de análisis Maxdiff}

Los técnicos de XLSTAT indicaron que rlh es la probabilidad de raíz (por Root Like Hood, en inglés). Este valor varía entre cero y uno, donde el valor de uno es un ajuste perfecto y el valor de cero implica que no hay ajuste (E. Anagnostou, correo electrónico, 4 de mayo del 2015). El modelo Maxdiff de esta investigación tiene un ajuste de 0.745 , lo que indica que sí hay un ajuste considerable para el modelo (E. Anagnostou, correo electrónico, 22 de mayo del 2015).

Datos cuantitativos, cualitativos o mixtos.

\footnotetext{
${ }^{2}$ Nota Aclaratoria: En lo referente a margen de error y confianza, para esta investigación se ha utilizado el criterio citado(Gomez, 2011), según el cuallos estudios deCiencias Sociales deben tener un $95 \%$ de confianza y un error ubicado entre el $4 \%$ y el $6 \%$,
} 
La investigación cualitativa aporto con 43 atributos relevantes, que según el cliente institucional deben poseer un cosmético antiedad de ojos. A estos atributos se los ha clasificado en 12 grupos, por características afines según lo expuesto en la tabla 2.

Tabla 2. Tabla de agrupación de atributos en macro-atributos

\begin{tabular}{|c|c|c|c|c|c|c|c|c|c|c|c|}
\hline drenador & refrescante & con antioxidantes & $\begin{array}{l}\text { precio } \\
\text { alto }\end{array}$ & crédito & $\begin{array}{l}\text { frasco de } \\
\text { vidrio }\end{array}$ & natural & $\begin{array}{c}\text { no probado } \\
\text { con animales }\end{array}$ & $\begin{array}{l}\text { aplicable con } \\
\text { aparatología }\end{array}$ & $\begin{array}{c}\text { grado } \\
\text { farmacéutico }\end{array}$ & $\begin{array}{c}\text { ingredientes } \\
\text { europeos }\end{array}$ & $\begin{array}{l}\text { capacitación } \\
\text { a cliente } \\
\text { corporativo }\end{array}$ \\
\hline reafirmante & hiperhemiante & descontracturante & & descuentos & $\begin{array}{c}\text { presentación } \\
\text { en tubo }\end{array}$ & antialérgico & & $\begin{array}{c}\text { fácil de } \\
\text { dosificar }\end{array}$ & & & \\
\hline \multirow[t]{9}{*}{$\begin{array}{c}\text { anti- } \\
\text { inflamatorio }\end{array}$} & & efecto tensor & & $\begin{array}{c}\text { disponibilidad } \\
\text { permnente }\end{array}$ & $\begin{array}{c}\begin{array}{c}\text { presentación } \\
\text { de lujo }\end{array} \\
\end{array}$ & $\begin{array}{c}\mathrm{no} \\
\text { comedogénico }\end{array}$ & & $\begin{array}{l}\text { que no haya } \\
\text { desperdicio }\end{array}$ & & & \\
\hline & & $\begin{array}{c}\text { formador de } \\
\text { colágeno }\end{array}$ & & $\begin{array}{c}\text { entrega a } \\
\text { local }\end{array}$ & & no graso & & extensible & & & \\
\hline & & rellena arrugas & & $\begin{array}{c}\text { fácil de } \\
\text { encontrar }\end{array}$ & & no irritante & & eranescente & & & \\
\hline & & hidratante & & promociones & & ph neutro & & & & & \\
\hline & & humec-tante & & $\begin{array}{c}\text { registro } \\
\text { sanitario }\end{array}$ & & sin ácidos & & & & & \\
\hline & & & & & & $\begin{array}{c}\text { sin fragancia } \\
\text { sintética } \\
\end{array}$ & & & & & \\
\hline & & & & & & \begin{tabular}{|c|}
$\begin{array}{c}\text { sin protector } \\
\text { solar }\end{array}$ \\
\end{tabular} & & & & & \\
\hline & & & & & & $\begin{array}{c}\text { sustancias } \\
\text { nutritivas }\end{array}$ & & & & & \\
\hline & & & & & & $\begin{array}{c}\text { todo tipo de } \\
\text { piel }\end{array}$ & & & & & \\
\hline $\begin{array}{c}\text { elimina las } \\
\text { bolsas de } \\
\text { ojos }\end{array}$ & $\begin{array}{c}\text { suprime } \\
\text { ojeras }\end{array}$ & erradica arrugas & $\begin{array}{c}\text { precio } \\
\text { alto }\end{array}$ & $\begin{array}{c}\text { beneficios } \\
\text { comerciales, } \\
\text { crédito y } \\
\text { promociones }\end{array}$ & presentación & $\begin{array}{c}\text { amigable con } \\
\text { la piel }\end{array}$ & ecológico & $\begin{array}{l}\text { fácil de } \\
\text { aplicar }\end{array}$ & $\begin{array}{l}\text { calidad grado } \\
\text { farmacéutico }\end{array}$ & $\begin{array}{c}\text { ingredientes } \\
\text { europeos }\end{array}$ & $\begin{array}{c}\text { capacitación a } \\
\text { cliente } \\
\text { corporativo }\end{array}$ \\
\hline
\end{tabular}

Nota. Fuente: Investigación cualitativa propia del autor.

En el área gris están contenidos los atributos básicos que, según los entrevistados, debe tener un contorno de ojos; en el área amarilla están los macro-atributos, ordenados de acuerdo a características similares de los atributos. Datos obtenidos por muestreo no probabilístico, a partir de 12 Unidades muéstrales.

La investigación cuantitativa se apoyó en los 12 atributos básicos obtenidos a través de la investigación cualitativa, para con estos atributos proceder a realizar el análisis MAXDIFF.

Los resultados numéricos medidos en porcentaje del análisis Maxdiff se encuentran en la Tabla 3. El atributo más relevante para la categoría de "cosméticos antiedad" es "erradica arrugas", seguido por el atributo "amigable con la piel". El atributo "precio bajo" no es relevante en el nicho de centros de estética del Distrito Metropolitano de Quito, y se ubica en el sexto lugar, pero sí es relevante el atributo "beneficios comerciales, créditos y descuentos", que se ubica en una tercera posición.

Tabla 3. Puntuaciones Maxdiff de atributos de contornos antiedad 


\begin{tabular}{|l|c|c|c|c|}
\hline \multicolumn{1}{|c|}{ Fuente } & Minimo & Máximo & Media & Desv. Tipica \\
\hline Erradica arrugas & 4,16 & 25,710 & 20,319 & 5,010 \\
\hline Amigable con la piel & 5,114 & 23,991 & 18,39 & 5,186 \\
\hline Beneficios comerciales, créditos y descuentos & 0,049 & 23,544 & 14,618 & 7,397 \\
\hline Ingredientes Europeos & 1,182 & 20,355 & 11,703 & 5,746 \\
\hline Calidad Farmacéutica & 0,005 & 19,953 & 7,896 & 7,563 \\
\hline Precio bajo & 2,52 & 15,430 & 7,574 & 3,753 \\
\hline Suprime ojeras & 0,000 & 22,351 & 5,146 & 7,549 \\
\hline Elimina las bolsas de ojos & 1,632 & 15,908 & 4,637 & 2,667 \\
\hline Capacitación a cliente corporativo & 0,987 & 15,180 & 4,319 & 3,338 \\
\hline Facilidad de empleo & 0,027 & 18,302 & 3,911 & 4,629 \\
\hline Presentación de lujo & 0,011 & 10,097 & 1,488 & 2,887 \\
\hline
\end{tabular}

Nota. Fuente: Investigación cuantitativa propia del autor. Tabla realizada con el software estadístico XLSAT 2015

\section{Análisis de datos con su debida interpretación}

En el gráfico de superficie de la figura 1 se puede observar por un método gráfico la importancia e interrelación de los diferentes atributos críticos de éxito para la categoría, en donde claramente predomina por área y altura color rojo correspondiente el atributo "erradica arrugas", ocupando el segundo lugar en importancia el atributo "amigable con la piel" en color purpura, para finalmente estar en tercer lugar el atributo "beneficios comerciales, créditos y " descuentos en tercer lugar, junto estos tres atributos suman una importancia relativa del $53.327 \%$. Implicado que el atributo el atributo más atractivo si bien es el más importante no está muy distanciado estadísticamente del segundo o el tercer atributo en importancia, implicando este dato que el atributo "erradica arrugas" debe utilizarse para estrategias de marketing o de posicionamiento junto con el atributo "amigable con la Piel" o B2beneficios comerciales, créditos y descuentos". En la tabla 3 se puede ver que el atributo con menos puntaje es "presentación de lujo" con un porcentaje del 1,488. Denotando que no es conveniente promocionar este atributo en el nicho de mercado de esta investigación, pues solo se estarían desperdiciando recursos de marketing, lo mismo para los atributos "facilidad de empleo", "capacitación cliente corporativo" y "elimina las bolsas de los ojos" y suprime ojeras. En cuanto al atributo precio, se puede mencionar que si bien no es el menos importante para el cliente corporativo de centros de estética, tampoco es el primordial, por lo que se sugiere no se deben hacer promociones de un producto antiedad de ojos basadas en el "precio bajo", pero si basadas en "beneficios comerciales, crédito y descuentos". 


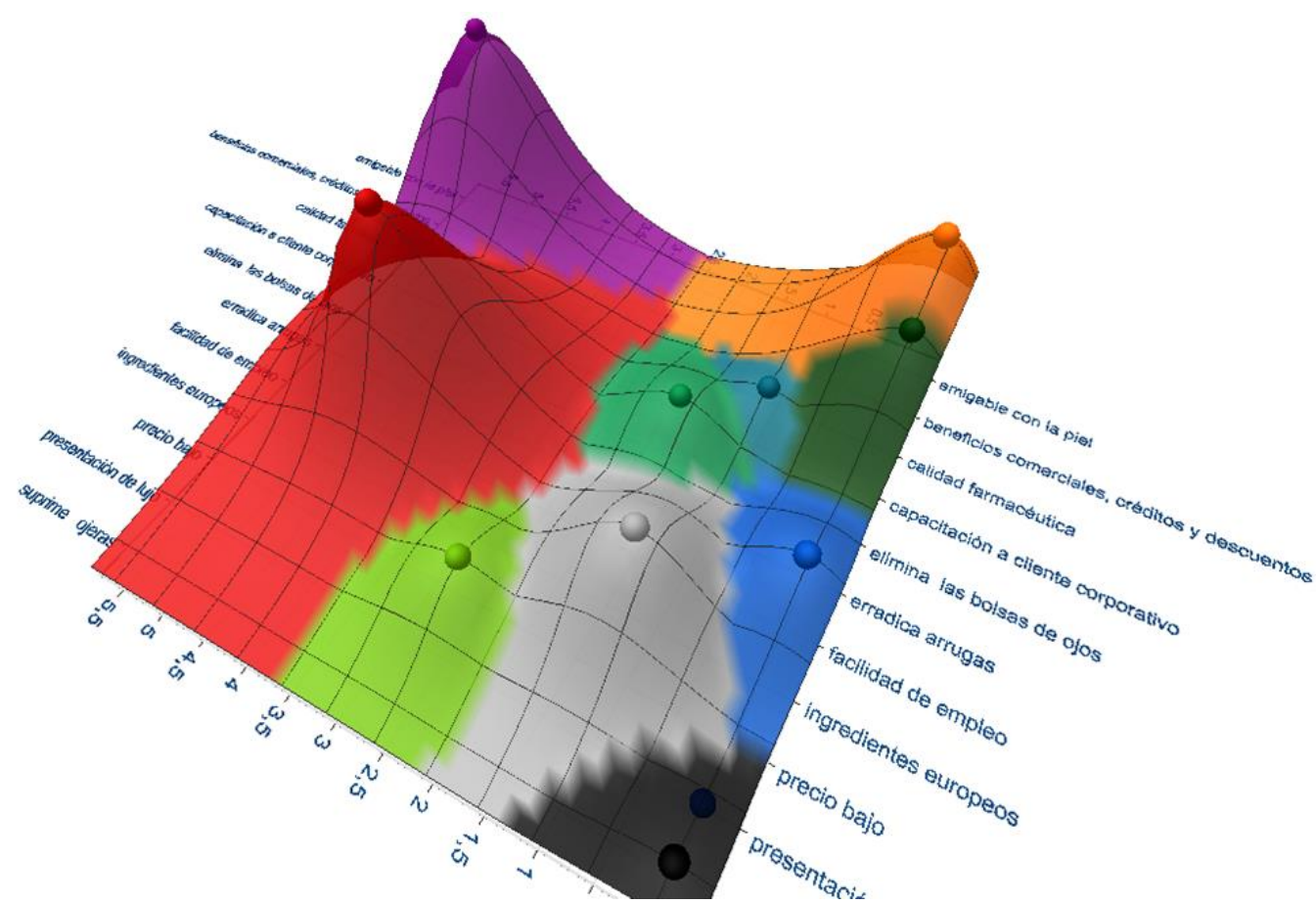

Figura 1. Gráfico 3dPlot Maxdiff de puntuaciones de atributos de cosméticos antiedad realizado con el software 3D Surface Plotting Tool. Fuente: Investigación cuantitativa, con un nivel de confianza del 95\% y un margen de error del 6\%, para un total de 96 unidades encuestadas con el método Hierarchical Bayes y una Convergencia del 0.0001.

Nota: Rojo Erradica arrugas, Purpura Amigable con la piel, Amarillo Beneficios comerciales, Verde calidad farmacéutica, Blanco Ingredientes europeos, Verde Flex precio bajo, Plomo suprime ojeras, Negro presentación de lujo

\section{Conclusiones}

En base a todo lo desarrollado en el estudio se obtuvieron las siguientes:

La propuesta de marketing estratégico de productos antiedad para ojos en el segmento estudiado debe estar relacionada principalmente con los atributo funcional "erradica arrugas"

El atributo funcional "erradica arrugas", es compatible para las estrategias de posicionamiento y diferenciación de marketing, con el atributo físico "amigable con la piel" o el atributo utilitario "beneficios comerciales" el cual posee una desviación típica de 7.397, respecto a su media de 14.618 lo que implica que hay clientes que valoran este atributo tanto como el atributo principal "erradica arrugas"

No se deben desperdiciar recursos de la empresa en lo referente a mercadeo de contornos de ojos antiedad en capacitaciones continuas que son muy poco valoradas por el cliente institucional (atributo capacitación).

El atributo precio bajo no es importante en el cliente institucional, ya que ocupa el sexto lugar en orden de importancia estadística de los atributos de más valorados por el segmento investigado 
El cliente institucional no valora que el producto sea ecológico, lo que más le importa es el atributo funcional de erradica arrugas.

Definitivamente no conviene apalancar ninguna estrategia de posicionamiento o de diferenciación, con el atributo presentación de lujo, por ocupar el lugar más bajo en el orden de importancia de los atributos.

Esta misma metodología se puede utilizar para cualquier tipo de producto o servicio, para poder descubrir el atributo más valorado por un mercado en específico.

\section{Significado de los resultados y/o su aplicación}

El resultado es claro, el factor más importante para un cosmético antiedad en el mercado institucional del Distrito Metropolitano de Quito, es el atributo "erradica arrugas", esto implica que es el atributo preferido por el nicho, haciendo notar que este es un atributo funcional sobre el que hay que realizar toda estrategia de Marketing, Posicionamiento y Promoción, sin embargo no implica que es el único atributo a ser considerado, por ejemplo en el momento de la venta, en un momento de la verdad, se pueden destacar atributos como "beneficios comerciales, crédito y descuentos" o "amigable con la piel"

La aplicación de este conocimiento implica el invertir los recursos de las empresas que se dedican a la venta de cosméticos de ojos en promociones relacionadas con los tres primeros atributos de la tabla 3 y no en los últimos, esto hará que haya se utilicen los recursos de marketing de una forma óptima y no se desperdicien en atributos poco importantes.

\section{Sugerencias para investigaciones futuras en relación al tema}

La metodología aplicada en este estudio tiene gran valor pedagógico, por lo que se siguiere enseñarla a estudiantes de Marketing, Planificación Estratégica, Estadística, y ramas afines para descubrir cuál es el atributo más importante de un producto y en base a ese atributo trabajar las distintas propuestas mercadológicas, también se sugiere hacer un estudio similar en otra ciudad ecuatoriana para comparar resultados y similitudes de agrado de atributos de cosméticos antiedad de ojos.

\section{Bibliografía}

El Comercio. (22 de julio de 2012). En el país hay 627 centros de estética.

Hair, J. (1999). Análisis Multivariante. México: Prentice Hall.

Martín Castellon, P., Lafuente Lechuga, M., \& Faura Matínez, U. (2015). Guía práctica de estadística aplicada al marketing y la empresa. Madrid: Paraninfo.

Ochoa, C. (2014). La actualidad sobre la investigación por internet. Recuperado el 3 de mayo de 2015, de http://www.netquest.com/blog/es/maxdiff/ 
Pérez, C. (2009). Técnicas Estadísticas Multivariantes con SPSS. Madrid: Garceta.

Sreedhar, G. (2016). Design Solutions for Improving Website Quality and Effectiveness. (I. P. R., Trans.) Hershey: Information Sciencie Reference.

Talledo, H. (3 de mayo de 2015). Técnicas y Herramientas para La Investigación De Mercados. Obtenido de http://es.slideshare.net/hernantalledo/tcnicas-y-herramientas-para-lainvestigacin-de-mercados-htalledo

Thompson, I. (26 de marzo de 2015). Marketing-free.com. Obtenido de http://www.marketingfree.com/producto/definicion-producto.html

Villaseca, D. (2014). Innovación y marketing de servicios en la era digital. Madrid: ESIC.

XLSTAT. (2015). MaxDiff analysis. Recuperado el 2 de mayo de 2015, de http://www.xlstat.com/es/productos-solutiones/caracteristica/max-diff-analysis.html 